hep-th/0308001

PUPT-2092

\title{
Singularities of $\mathcal{N}=1$ Supersymmetric Gauge Theory and Matrix Models
}

\author{
David Shih \\ Department of Physics, Princeton University, Princeton, NJ 08544
}

\begin{abstract}
In $\mathcal{N}=1$ supersymmetric $U(N)$ gauge theory with adjoint matter $\Phi$ and polynomial tree-level superpotential $W(\Phi)$, the massless fluctuations about each quantum vacuum are generically described by $U(1)^{n}$ gauge theory for some $n$. However, by tuning the parameters of $W(\Phi)$ to non-generic values, we can reach singular vacua where additional fields become massless. Using both the matrix model prescription and the strong-coupling approach, we study in detail three examples of such singularities: the singularities of the $n=1$ branch, intersections of $n=1$ and $n=2$ branches, and a class of $\mathcal{N}=1$ ArgyresDouglas points. In all three examples, we find that the matrix model description of the low-energy physics breaks down in some way at the singularity.
\end{abstract}

August, 2003 


\section{Introduction}

Following the work of Dijkgraaf and Vafa [1] 3], much recent progress has been made in the study of a wide class of supersymmetric gauge theories and their connections to bosonic matrix models. Probably the simplest and most well-studied example in this context (see e.g. [4[10]) has been $\mathcal{N}=1$ supersymmetric $U(N)$ gauge theory, with matter $\Phi$ in the adjoint representation and polynomial tree-level superpotential

$$
W(\Phi)=\sum_{r=0}^{k} \frac{g_{r}}{r+1} \operatorname{Tr} \Phi^{r+1}
$$

The associated matrix model in this case is a zero-dimensional model of an $\widehat{N} \times \widehat{N}$ complex matrix $M$, with potential $W(M)$. The idea of [1] was that the $\widehat{N} \rightarrow \infty$ limit of the matrix model could be used to obtain certain exact, non-perturbative gauge theory quantities, in a low-energy description of the gauge theory that we will call the "glueball description." It will be useful to briefly review the semiclassical arguments that lead to the glueball description. For generic tree-level superpotential, $\Phi$ acquires a mass and an expectation value, breaking $U(N)$ down to $G=\Pi_{i=1}^{n} U\left(N_{i}\right) \cong U(1)^{n} \times \Pi_{i=1}^{n} S U\left(N_{i}\right)$ for some $n \leq k$. Integrating out $\Phi$ and the massive $W$-bosons leads to an effective theory of pure superYang-Mills with gauge group $G$. At lower energies, the $S U\left(N_{i}\right)$ factors are believed to confine and become massive, leaving behind only a $U(1)^{n}$ gauge theory in the far infrared. However, it is also believed that the glueball fields $S_{i}$ of the $S U\left(N_{i}\right)$ factors behave as elementary fields in the IR [11]. These $n$ massive glueball fields together with the $U(1)^{n}$ photons comprise the glueball description of the low-energy gauge theory.

The quantities computed by the associated matrix model are the effective superpotential $W_{\text {eff }}\left(S_{i}\right)$ for the $n$ glueball fields and the matrix of low-energy $U(1)^{n}$ gauge couplings. The effective glueball superpotential is the source of much interesting physics. For instance, extremizing $W_{\text {eff }}$ leads to the exact, non-perturbative, quantum vacua of the original gauge theory. Also the quadratic part of $W_{e f f}$ indicates whether the $S_{i}$ are massive or not, if one also assumes regularity of the Kähler potential. For generic tree-level superpotentials, we expect the matrix model to provide a good description of the low-energy physics, in the sense that it reproduces the exact non-perturbative vacuum structure and gives the correct massless spectrum (a number of $U(1)$ multiplets).

In this paper, we will be interested in how the generic picture can be modified at strong-coupling singularities where additional fields become massless. These singularities 
are reached by tuning one or more parameters of $W(\Phi)$ to special values, and are thus nongeneric. In the following sections, we will study some examples of such singularities. The examples are certainly worth studying for their own sake, as the presence of extra massless fields can lead to novel low-energy physics such as massless monopoles and interacting SCFTs. In addition, the study of singularities will teach us about the limitations of the gauge theory/matrix model correspondence, since we expect the glueball description to break down at singularities. The reason for this is that the extra fields that become massless at a singularity can be thought of as fields (i.e. monopoles and the Casimirs $\operatorname{Tr} \Phi^{k}$ ) in the "strong-coupling description" of the low-energy theory [12, 13] based on the exact Seiberg-Witten solution of the underlying $\mathcal{N}=2$ gauge theory 14 -18 1 . Thus they are not included in the glueball description. Rather, they have been integrated out, and integrating out massless fields usually leads to a breakdown in an effective theory. Through our study of examples, we will see various ways in which the breakdown of the glueball description is manifested. At the same time, it is important to keep in mind that the extrema of the effective glueball superpotential should still determine the vacua of the theory even when the glueball description itself breaks down. This is guaranteed by holomorphy.

The outline of the paper is as follows. We study the three examples described below in sections 2-4, respectively. Appendix A contains some calculations relevant to the study of the $n=1 / n=2$ singularity in section 2. Appendix $\mathrm{B}$ describes the matrix model calculation of the effective glueball superpotential relevant to the discussion of the ArgyresDouglas $(\mathrm{AD})$ point in section 3. The three examples we will study are:

1. Singularities of the $n=1$ branch. We will study the $n=1$ branch for tree-level superpotential of arbitrary degree, generalizing the results of [8]. We will show that the only singularities are those at which $\operatorname{Tr} \Phi$ becomes massless in the strong-coupling description. At such points, the mass of the glueball field $S$ also goes to zero, indicating a breakdown of the glueball description. We will also discuss other pathologies of the glueball description where the effective glueball superpotential becomes non-analytic. This is taken to be evidence that the glueball description has, in some sense, a finite

1 We will assume that the strong-coupling description is applicable even at singularities. This is possible for the following reason. If we write $W^{\prime}(x)=g \prod_{i=1}^{k}\left(x-a_{i}\right)$, then the strong-coupling approach is always valid for $g$ sufficiently small. Meanwhile, singular behavior is generally controlled by the values of the $a_{i}$. Therefore, we can tune the $a_{i}$ to reach a singularity, while keeping $g$ sufficiently small to ensure that the strong-coupling description remains valid. 
radius of convergence. We argue that at such pathological points, the Kähler potential for $S$ must also degenerate.

2. Intersections of $n=1$ and $n=2$ branches for cubic tree-level superpotential. These singularities were first considered in [5,8]. They occur when a vacuum with unbroken gauge group $U\left(N_{1}\right) \times U\left(N_{2}\right)$ meets a vacuum with unbroken gauge group $U(N)$. The singularity arises from an additional monopole becoming massless in the strongcoupling description. We will solve the glueball equations of motion exactly at the $n=1 / n=2$ singularity, which will lead to general formulas (functions of $N_{1}$ and $N_{2}$ ) for the parameters of $W(\Phi)$ and the vevs of chiral operators at these singularities. Previously, the $n=1 / n=2$ singularities were studied only for examples of $U(3)$ $U(6)$ in [5]. Our general analysis significantly extends these results. In the process, we confirm that extremizing the effective glueball superpotential leads to the correct vacua even at singularities where the glueball description breaks down. We also show that the breakdown of the glueball description at the $n=1 / n=2$ singularity occurs through a logarithmic divergence in the low-energy gauge coupling. This divergence is to be expected from the strong-coupling description, where a charged monopole field becomes massless.

3. $\mathcal{N}=1$ Argyres-Douglas points with $W(\Phi)=\operatorname{Tr} \Phi^{n+1}$. Here, the singularity results from a number of mutually non-local monopoles becoming massless. We will focus on the case $n=n_{\text {min }}=\left[\frac{N+1}{2}\right] ; \mathcal{N}=1 \mathrm{AD}$ points with $n>n_{\text {min }}$ have been studied recently in [19,20]. (The cases with $n<n_{\text {min }}$ are uninteresting, since they does not lead to $\mathcal{N}=1 \mathrm{AD}$ points.) There has been some question as to whether the points with $n=n_{\text {min }}$ are actually singular. We will compute the effective superpotential in both the glueball and the strong-coupling descriptions in an attempt to answer this question. We will confirm that for $N$ even, the points with $n=n_{\min }$ are non-singular; while for $N$ odd we will find evidence that these points lead to an interacting IR SCFT . The evidence takes the form of a non-analytic strong-coupling superpotential. Interestingly, the glueball superpotential remains analytic, although a glueball becomes massless. Thus, as in the singularities of the $n=1$ branch, a massless glueball again signifies a breakdown in the glueball description due to the presence of additional massless fields. However in this case, the massless fields are mutually non-local monopoles. 


\section{Singularities of the $n=1$ branch}

The first set of singularities we will study are those of the $n=1$ branch. These singularities were previously discussed in [8] for cubic tree-level superpotential. Our results generalize the discussion to superpotentials of arbitrary degree, and also hopefully clarify some points of confusion. We follow the approach and conventions of [4]. Let the tree-level superpotential be given by (1.1). For a given $W(\Phi)$, there are many quantum vacua, each associated with a choice of semi-classical gauge group $\Pi_{i} U\left(N_{i}\right)$. The vacua corresponding to unbroken gauge group $U(N)$ sweep out the $n=1$ branch as the parameters of $W(\Phi)$ are varied. On the $n=1$ branch, there is only one glueball field $S$ in the glueball description. Similarly, in the strong coupling description there is only one independent field $z_{0}=\frac{1}{N} \operatorname{Tr} \Phi$ remaining after the $N-1$ condensed monopoles have been integrated out. The effective superpotential for $z_{0}$ can be obtained exactly with the help of Chebyshev polynomials [21]. The result is [4]:

$$
W_{e f f}\left(z_{0}\right)=N \sum_{l=0}^{\left[\frac{k+1}{2}\right]} \frac{\Lambda^{2 l}}{(l !)^{2}} W^{(2 l)}\left(z_{0}\right)
$$

The vev of $z_{0}$ is determined by solving the following polynomial equation for $z_{0}$ :

$$
W_{e f f}^{\prime}\left(\left\langle z_{0}\right\rangle\right)=N \sum_{l=0}^{\left[\frac{k+1}{2}\right]} \frac{\Lambda^{2 l}}{(l !)^{2}} W^{(2 l+1)}\left(\left\langle z_{0}\right\rangle\right)=0 .
$$

Since $W_{\text {eff }}\left(z_{0}\right)$ is polynomial in $z_{0}$, it is always finite and regular near its extrema. Therefore, assuming regularity of the Kähler potential (which is a valid assumption at least for $W(\Phi)$ sufficiently small), the only possible singularities on the $n=1$ branch must occur at points where $z_{0}$ becomes massless. The condition for massless $z_{0}$ is simply

$$
M_{0} \equiv W_{e f f}^{\prime \prime}\left(\left\langle z_{0}\right\rangle\right)=N \sum_{l=0}^{\left[\frac{k-1}{2}\right]} \frac{\Lambda^{2 l}}{(l !)^{2}} W^{(2 l+2)}\left(\left\langle z_{0}\right\rangle\right)=0 .
$$

We can think of (2.3) as a restriction on the form of the tree-level superpotential. The tree-level superpotential originally has $k+1$ parameters, $\left(g_{0}, \ldots, g_{k}\right)$. After using (2.2) to write $\left\langle z_{0}\right\rangle$ in terms of the $g_{i}$, (2.3) becomes one equation relating these parameters. Therefore the massless $z_{0}$ singularities comprise a $k$ dimensional subspace of the $k+1$ dimensional parameter space of tree-level superpotentials.

Now let us see how the massless $z_{0}$ singularities are realized in the glueball description. Treating the glueball field as an elementary degree of freedom in the IR depends on being 
able to integrate out all the components of the adjoint field $\Phi$, including $z_{0}$. Thus when $z_{0}$ is massless, the glueball analysis should break down somehow. The effective glueball superpotential can be obtained through a Legendre transform of (2.1) with respect to $2 N \log \Lambda$ [4]. The result of the Legendre transform is an effective superpotential for $S, z_{0}$ and an auxiliary field $C$ :

$$
W_{e f f}\left(S, C, z_{0}\right)=2 N S \log \left(\frac{\Lambda}{C}\right)+N \sum_{l=0}^{\left[\frac{k+1}{2}\right]} \frac{C^{2 l}}{(l !)^{2}} W^{(2 l)}\left(z_{0}\right) .
$$

Integrating out $C$ and $S$ using their equations of motion returns us to (2.1), while integrating out $C$ and $z_{0}$ gives the effective glueball superpotential. The equations of motion for $C$ and $z_{0}$ are:

$$
\begin{aligned}
& \sum_{l=0}^{\left[\frac{k+1}{2}\right]} \frac{C^{2 l}}{(l !)^{2}} W^{(2 l+1)}\left(z_{0}\right)=0 \\
& \sum_{l=1}^{\left[\frac{k+1}{2}\right]} \frac{l C^{2 l}}{(l !)^{2}} W^{(2 l)}\left(z_{0}\right)=S .
\end{aligned}
$$

Solving for $C$ and $z_{0}$ and substituting back into (2.4) results in an effective superpotential for $S$, i.e.

$$
W_{e f f}(S)=W_{e f f}\left(S, C(S), z_{0}(S)\right)
$$

with $C(S)$ and $z_{0}(S)$ determined implicitly via (2.5).

While it is not obvious that there exists a closed-form expression for $W_{\text {eff }}(S)$, one can easily show that the first derivative of $W_{\text {eff }}(S)$ is given by the very simple expression

$$
W_{e f f}^{\prime}(S)=2 N \log \left(\frac{\Lambda}{C(S)}\right) \text {. }
$$

Moreover, using (2.5), one can show that the second derivative of $W_{\text {eff }}$ is

$$
W_{e f f}^{\prime \prime}(S)=\frac{N A}{B^{2}-C^{2} A^{2}}
$$

with

$$
\begin{aligned}
& A=\sum_{l=0}^{\left[\frac{k-1}{2}\right]} \frac{C^{2 l}}{(l !)^{2}} W^{(2 l+2)}\left(z_{0}\right) \\
& B=\sum_{l=1}^{\left[\frac{k+1}{2}\right]} \frac{l C^{2 l}}{(l !)^{2}} W^{(2 l+1)}\left(z_{0}\right) .
\end{aligned}
$$


From (2.7), we see that the extrema of the effective superpotential occur when $C(S)=\Lambda$. Substituting this in (2.9) and comparing with (2.3), we see that $N\langle A\rangle=M_{0}$. Thus at an extremum, (2.8) becomes

$$
W_{e f f}^{\prime \prime}(\langle S\rangle)=\frac{N^{2} M_{0}}{N^{2}\langle B\rangle^{2}-\Lambda^{2} M_{0}^{2}} .
$$

It is clear from (2.10) that the glueball $S$ can become massless only when the mass $M_{0}$ of $z_{0}$ also goes to zero. Examples of such singularities for the case of cubic superpotential $k=2$ were discussed in [8]. Our general analysis makes it clear that there are no genuinely massless glueball points: since the fundamental field $z_{0}$ also becomes massless, it is incorrect to treat the composite glueball field as an elementary excitation of the low-energy theory. Rather, we should think of the massless glueball as a sign that the glueball description has broken down.

We should also consider the possibility that the denominator of (2.10) vanishes. This pathological behavior can occur for either $z_{0}$ massive or massless, and it can result in an "infinite mass" for the glueball field. But the fact that it is not correlated with the mass of $z_{0}$, together with the fact that the strong-coupling description in terms of $z_{0}$ is comparatively well-behaved, suggests that these pathological points should not be thought of as additional singularities. Rather, we should interpret them as evidence for the finite radius of convergence of the glueball description. At such points it is reasonable to suppose that the Kähler potential for $S$ also degenerates in such a way that the entire pathology can be removed with a field redefinition, so that the redefined glueball field is massive. With this plausible assumption, the only true singularities of the low-energy theory where additional fields become massless are the massless $z_{0}$ points.

\section{The $n=1 / n=2$ singularity}

\subsection{Solving the glueball equations of motion}

The second class of singularities we will study are the intersections of the $n=1$ and $n=2$ branches. These singularities occur at special values of the tree-level superpotential parameters where a vacuum with unbroken gauge group $U\left(N_{1}\right) \times U\left(N_{2}\right)$ meets a vacuum with unbroken gauge group $U(N)$. The basic features of such singularities were discussed in [5] using the strong-coupling approach. In this section, we will study the intersection singularity in greater detail using the glueball description. We will focus on studying the 
approach to the singularity from the $n=2$ branch, because the corresponding approach from the $n=1$ branch generally exhibits no singular behavior. This can be seen in a variety of ways. For example, from the previous section we know that the only singularities of the $n=1$ branch are those associated with massless $z_{0}$. Alternatively, the matrix model curve on the $n=1$ branch is perfectly regular as we pass through the intersection with the $n=2$ branch: it always has a single branch cut.

We will take the tree-level superpotential to be cubic to simplify the calculations. The qualitative features of the analysis, however, should be common to $n=1 / n=2$ singularities for superpotentials of arbitrary degree. As we approach the $n=1 / n=2$ singularity from the $n=2$ branch, both the matrix model curve and the $\mathcal{N}=2$ curve acquire an extra double root. Using the matrix model curve, we can calculate the effective glueball superpotential and the matrix of $U(1)$ gauge couplings near the singularity. The matrix model curve takes the form

$$
y_{m}^{2}=\left(x^{2}-m^{2}\right)^{2}+f_{1} x+f_{0}=\left(x-r_{1}\right)\left(x-r_{2}\right)\left(x-r_{3}\right)\left(x-r_{4}\right) .
$$

Note that we have lost no generality by taking the tree-level superpotential to have the form $W^{\prime}(x)=x^{2}-m^{2}$. The most general cubic superpotential can always be recovered by a shift in $x$.

The first parametrization of the matrix model curve makes it clear that $m$ is a parameter and $f_{0}$ and $f_{1}$ are fluctuating fields related to the two glueball fields $S_{1}$ and $S_{2}$ by a field redefinition. However, the second parametrization in terms of the roots $r_{i}$ of $y_{m}$ will prove more convenient in the calculations to follow. We should think of the four $r_{i}$ as fields subject to the two constraints that the highest order terms in (3.1) be given by $W^{\prime}(x)^{2}$. We can always return to the first interpretation involving $f_{0}$ and $f_{1}$ by matching the two parameterizations of (3.1).

To compute the effective glueball superpotential, we must evaluate the period integrals of the matrix model curve. This results in a complicated and not very illuminating combination of elliptic integrals. Fortunately, however, the first derivatives with respect to $f_{j}$ - and hence the glueball equations of motion - are relatively simple to evaluate. The calculation is briefly outlined in appendix A; here we simply exhibit the final result 
(dropping as usual irrelevant terms of $\left.\mathcal{O}\left(\frac{1}{\Lambda_{0}}\right)\right)$ :

$$
\begin{aligned}
\frac{\partial W_{e f f}}{\partial f_{0}}= & \frac{1}{\sqrt{\left(r_{4}-r_{2}\right)\left(r_{3}-r_{1}\right)}}\left(N F(\theta \mid R)-N_{2} F\left(\frac{\pi}{2} \mid R\right)\right) \\
\frac{\partial W_{e f f}}{\partial f_{1}}= & \frac{1}{2} N \log \left(\frac{r_{4}+r_{3}-r_{2}-r_{1}}{4 \Lambda}\right)+\frac{r_{1}}{\sqrt{\left(r_{4}-r_{2}\right)\left(r_{3}-r_{1}\right)}}\left(N F(\theta \mid R)-N_{2} F\left(\frac{\pi}{2} \mid R\right)\right) \\
& \quad+\frac{\left(r_{2}-r_{1}\right)}{\sqrt{\left(r_{4}-r_{2}\right)\left(r_{3}-r_{1}\right)}}\left(N \Pi(n ; \theta \mid R)-N_{2} \Pi\left(n ; \frac{\pi}{2} \mid R\right)\right)
\end{aligned}
$$

where

$$
\sin ^{2} \theta=\frac{r_{4}-r_{2}}{r_{4}-r_{1}}, \quad R=\frac{\left(r_{3}-r_{2}\right)\left(r_{4}-r_{1}\right)}{\left(r_{3}-r_{1}\right)\left(r_{4}-r_{2}\right)}, \quad n=\frac{r_{3}-r_{2}}{r_{3}-r_{1}}
$$

and $F$ and $\Pi$ are the standard elliptic integrals of the first and third kinds, respectively. Their explicit definitions are given in appendix A.2

Eqn. (3.2) is very interesting, as it represents a general formula for the derivatives of the two-cut effective superpotential, valid for every choice of $N_{1}$ and $N_{2}$. Let us mention a couple of caveats, however. First, as mentioned in the footnote, we have assumed $b_{2}=0$ for simplicity; the effects of $b_{2} \neq 0$ can be easily included. Secondly, we have made the crucial ansatz that the $r_{i}$ lie on the real line. With this ansatz, we can assume without loss of generality that $r_{1}<r_{2}<r_{3}<r_{4}$, and we can choose the cuts of $y_{m}$ to be $\left(r_{1}, r_{2}\right)$ and $\left(r_{3}, r_{4}\right)$. This is the starting point of the calculations leading to (3.2) described in appendix A. Only with these assumptions about the $r_{i}$ are the elliptic integrals in (3.2) are unambiguously defined. One might hope to analytically continue (3.2) to arbitrary complex $r_{i}$, but this procedure probably suffers from various ambiguities. Perhaps with a little more effort these ambiguities can be brought under control, but we postpone this for future work. Fortunately, it is not relevant for the $n=1 / n=2$ singularity.

The equations of motion for $f_{0}$ and $f_{1}$ are obtained by setting the derivatives (3.2) to zero. We can rewrite them more simply as:

$$
\begin{aligned}
& N F(\theta \mid R)-N_{2} F\left(\frac{\pi}{2} \mid R\right)=0 \\
& N \log \left(\frac{r_{4}+r_{3}-r_{2}-r_{1}}{4 \Lambda}\right)=-\frac{2\left(r_{2}-r_{1}\right)}{\sqrt{\left(r_{4}-r_{2}\right)\left(r_{3}-r_{1}\right)}}\left(N \Pi(n ; \theta \mid R)-N_{2} \Pi\left(n ; \frac{\pi}{2} \mid R\right)\right) .
\end{aligned}
$$

2 We assume throughout this section that the integer $b_{2}=0$. The effect of nonzero $b_{2}$ is easily included by adding to (3.2) the formula for the derivatives of $S_{2}$ given in appendix A. In any case, a trivial calculation shows that $b_{2}=0$ near an $n=1 / n=2$ singularity. 
Notice that the equations of motion are most naturally expressed in terms of the roots $r_{i}$ of the matrix model curve. As discussed above, these roots are subject to two constraints:

$$
\begin{aligned}
& r_{1}+r_{2}+r_{3}+r_{4}=0 \\
& \left(r_{2}-r_{3}\right)^{2}+\left(r_{1}-r_{4}\right)^{2}-2\left(r_{2}+r_{3}\right)\left(r_{1}+r_{4}\right)=8 m^{2} .
\end{aligned}
$$

These constraints ensure that the highest-order terms in $y_{m}^{2}$ are given by $W^{\prime}(x)^{2}$. Combining (3.4) with (3.5) allows us to solve for the $r_{i}$ in terms of the parameters $N_{1}, N_{2}$ and $m$. Matching the two parameterizations of the matrix model curve (3.1) yields the expectation values of the fields $f_{0}$ and $f_{1}$.

Up till now our computation of $W_{\text {eff }}$ and the equations of motion has been quite general (modulo the caveats described above), valid not just near the $n=1 / n=2$ singularity. However, the equations of motion (3.4) are clearly quite forbidding, and a general solution is not readily apparent. Thus it comes as a rather pleasant surprise that they simplify dramatically at the $n=1 / n=2$ singularity! The source of the simplification is the fact that $r_{2}=r_{3}$ at an $n=1 / n=2$ singularity. (Recall that the cuts of $y_{m}$ were chosen to be $\left(r_{1}, r_{2}\right)$ and $\left(r_{3}, r_{4}\right)$, with the ansatz that the $r_{i}$ are real and $r_{1}<r_{2}<r_{3}<r_{4}$.) From (3.3), we see this implies that $n=R=0$. Then the elliptic integrals reduce to $F(\theta \mid 0)=\Pi(0 ; \theta \mid 0)=\theta$, and the equations of motion and constraints become:

$$
\begin{aligned}
& \sqrt{\frac{r_{4}-r_{2}}{r_{4}-r_{1}}}=\sin \left(\frac{\pi}{2} \frac{N_{2}}{N}\right) \\
& r_{4}-r_{1}=4 \eta \Lambda \\
& r_{1}+2 r_{2}+r_{4}=0 \\
& \left(r_{1}-r_{4}\right)^{2}-4 r_{2}\left(r_{1}+r_{4}\right)=8 m^{2} .
\end{aligned}
$$

Notice that since $r_{2}=r_{3}$, there are four equations for three unknowns, and thus for generic $m, N_{1}$ and $N_{2}$ there is no solution to the equations of motion. In order for there to be a solution, we must tune the parameter $m$ to special values depending on $N_{1}$ and $N_{2}$. Here $\eta$ is an $2 N$ th root of unity, which we must include in order to obtain all of the $n=1 / n=2$ singularities. It arises through the presence of the logarithm in (3.2) and (3.4), which should be thought of as a Veneziano-Yankielowicz-type term [11], in which case it is more properly written as $\log \left[\left(\frac{r_{4}+r_{3}-r_{2}-r_{1}}{4 \Lambda}\right)^{2 N}\right]$. The equations of motion for $r_{2}=r_{3}$ require this logarithm to be zero, resulting in the $2 N$ branches shown in (3.6) labelled by $\eta$. 
Solving (3.6) for $r_{i}$ and $m$, we obtain

$$
\begin{aligned}
& r_{1}=-\eta \Lambda\left(2+\cos \frac{\pi N_{2}}{N}\right) \\
& r_{2}=r_{3}=\eta \Lambda \cos \frac{\pi N_{2}}{N} \\
& r_{4}=\eta \Lambda\left(2-\cos \frac{\pi N_{2}}{N}\right) \\
& m^{2}=\eta^{2} \Lambda^{2}\left(2+\cos ^{2} \frac{\pi N_{2}}{N}\right) .
\end{aligned}
$$

Matching the two parameterizations of the matrix model curve (3.1), we obtain the expectation values of the fields $f_{0}$ and $f_{1}$ at the double-root singularity:

$$
\begin{aligned}
& \left\langle f_{0}\right\rangle=-4 \eta^{4} \Lambda^{4}\left(1+2 \cos ^{2} \frac{\pi N_{2}}{N}\right) \\
& \left\langle f_{1}\right\rangle=8 \eta^{3} \Lambda^{3} \cos \frac{\pi N_{2}}{N} .
\end{aligned}
$$

Using the fact that the total glueball field is related to $f_{1}$ via $S=-\frac{1}{4} f_{1}$, we also obtain the expectation value of the glueball field at the $n=1 / n=2$ singularity:

$$
\langle S\rangle=-2 \eta^{3} \Lambda^{3} \cos \frac{\pi N_{2}}{N} .
$$

The general formulas (3.7), (3.8) and (3.9) for the form of the matrix model curve, the parameters of the tree-level superpotential and the expectation value of the glueball field at the $n=1 / n=2$ singularity were not known before. Previously, the locations of the $n=1 / n=2$ singularities and the expectation value of $S$ at the singularities were obtained only for sufficiently small values of $N$ where the $\mathcal{N}=2$ factorization problem could be explicitly solved [5, 8]. The factorization problem grows in complexity with $N$, but by studying the glueball equations of motion at the $n=1 / n=2$ singularity, we have sidestepped the difficulty of the solving the general factorization problem and obtained the quantum vacua at the singularity for general $N_{1}, N_{2}$.

To illustrate the power of our general results, it will be useful to compare them with an explicit example from [5]. For the case of $U(3)$, the only possible choices of breaking pattern with $n=2$ are $\left(N_{1}, N_{2}\right)=(1,2)$ and $(2,1)$. (3.7) and (3.8) then predict that the matrix model curve takes the form

$$
y_{m}^{2}=\left(x^{2}-m^{2}\right)^{2}-4 \eta^{3} \Lambda^{3}(x+m)
$$


with $m=3 \eta \Lambda / 2$ and $\eta^{6}=1$. In [5], solving the factorization problem resulted in the matrix model curve $y_{m}^{2}=\left(x^{2}-a^{2}\right)^{2}-4 \epsilon \Lambda^{3}(x+a)$, and intersections with the $n=1$ branch occurred at $8 a^{3}=27 \epsilon \Lambda^{3}$. This is in precise agreement with the glueball approach.

One can similarly verify that the cases of $U(4), U(5)$ and $U(6)$ studied in [5] agree exactly with the predictions of the general formulas (3.7)-(3.9) derived here. When performing these checks, and in general when using the formulas of this section, it is important to remember that $N_{1}$ and $N_{2}$ are defined to be the on-shell periods of the one-form $T(x)$ at the $n=1 / n=2$ singularity (we refer the reader to [5] for the definition of $T(x)$ and the details). This must be distinguished from the various values of $\left(N_{1}, N_{2}\right)$ that can be realized via semiclassical $\Lambda \rightarrow 0$ limits on a given branch of vacua. For instance, in the case of $U(4)$, the confining branches have unbroken gauge group $U(2) \times U(2)$ semiclassically, while the Coulomb branches have two different semiclassical limits corresponding to unbroken gauge group $U(3) \times U(1)$ and $U(2) \times U(2)$. In the general formulas of this section one must use $\left(N_{1}, N_{2}\right)=(2,2)$ for the confining branches and $\left(N_{1}, N_{2}\right)=(3,1)$ for the Coulomb branches in order to compare with [5], since these are the values of $\left(N_{1}, N_{2}\right)$ at the $n=1 / n=2$ singularities of these branches. 3

Having solved the equations of motion and obtained the vacua at the $n=1 / n=2$ singularity, it is not difficult to take the calculation off-shell, i.e. expand around these vacua and obtain the effective glueball superpotential near the singularity. This can be done, for instance, by expanding the first derivatives (3.2) of $W_{\text {eff }}$ in powers of $f_{0}-\left\langle f_{0}\right\rangle$ and $f_{1}-\left\langle f_{1}\right\rangle$ and then integrating. After a somewhat lengthy calculation, one finds that the effective glueball superpotential is regular at the singularity when written in terms of $f_{0}$ and $f_{1}$, and all fluctuations are massive. This agrees with the expectation from the strong-coupling analysis that the $n=1 / n=2$ singularity arises solely from a charged monopole becoming massless, with all other fields massive.

\subsection{The matrix of coupling constants near the singularity}

In order to see from the glueball analysis the effect of the monopole becoming massless at the singularity, we need to compute the matrix of gauge couplings $\tau_{i j}$ near the singularity. We will see that $\tau_{i j}$ tends to zero as an inverse logarithm as we approach the singularity. This will indicate that an additional charged field is becoming massless. Notice, however, that the glueball description fails to provide an explanation for this massless, charged

3 We thank C. Ahn for bringing this point to our attention. 
field. For that, we need the strong-coupling description, in which this new massless field is a monopole. Moving along the $n=2$ branch, the monopole acquires a bare mass, while moving along the $n=1$ branch condenses the monopole, spontaneously breaking $U(1)^{2}$ down to $U(1)$ via the Higgs mechanism.

The matrix of gauge couplings is given by the formula [4:

$$
\frac{1}{2 \pi i} \tau_{i j}=\frac{\partial \Pi_{i}}{\partial S_{j}}-\delta_{i j} \frac{1}{N_{i}} \sum_{l=1}^{n} N_{l} \frac{\partial \Pi_{i}}{\partial S_{l}}
$$

For $n=2$, this simplifies to

$$
\tau_{i j}=\left(\begin{array}{ll}
\tau_{11} & \tau_{12} \\
\tau_{21} & \tau_{22}
\end{array}\right)=2 \pi i \frac{\partial \Pi_{2}}{\partial S_{1}}\left(\begin{array}{cc}
-\frac{N_{2}}{N_{1}} & 1 \\
1 & -\frac{N_{1}}{N_{2}}
\end{array}\right),
$$

where we have used the fact that $\frac{\partial \Pi_{1}}{\partial S_{2}}=\frac{\partial \Pi_{2}}{\partial S_{1}}$ since $\Pi_{i}$ is itself a derivative $\frac{\partial \mathcal{F}}{\partial S_{i}}$ of the prepotential. Thus we see that the matrix of coupling constants depends only on the single derivative $\frac{\partial \Pi_{2}}{\partial S_{1}}$, evaluated the extremum of the effective superpotential. In appendix A we calculate this partial derivative; it can be related to the derivatives with respect to $f_{0}$ and $f_{1}$ via the chain rule. The answer, to leading order in $R$, is simply

$$
\frac{\partial \Pi_{2}}{\partial S_{1}}=\frac{\pi i N_{1} N_{2}}{N^{2}} \frac{1}{\log \left(\frac{16}{R}\right)}+\mathcal{O}(R)
$$

As we approach the singularity, we see that $\tau_{i j}$ has the limiting behavior:

$$
\frac{1}{2 \pi i} \tau_{i j}=\frac{\pi i N_{1} N_{2}}{N^{2}} \frac{1}{\log \left(\frac{16}{R}\right)}\left(\begin{array}{cc}
-\frac{N_{2}}{N_{1}} & 1 \\
1 & -\frac{N_{1}}{N_{2}}
\end{array}\right)+\mathcal{O}(R) .
$$

Therefore $\tau_{i j} \rightarrow 0$ as an inverse logarithm as we approach the $n=1 / n=2$ singularity. This means that $\tau_{i j}$ is continuous as we pass from the $n=2$ branch to the $n=1$ branch, since $\tau_{i j}=0$ trivially on the $n=1$ branch. More importantly, however, the logarithm indicates that the gauge coupling constant of the non-trivial $U(1)$, given roughly by $1 / \tau$, diverges as we approach the singularity. The divergence is due to an additional monopole becoming massless at the singularity, which causes an IR divergence in the renormalized coupling of the $U(1)$ under which it is charged. 


\section{4. $\mathcal{N}=1$ Argyres-Douglas points}

The third and final set of singularities we will consider are the $\mathcal{N}=1$ AD points [22,26]. Such points are believed to represent a novel class of interacting $\mathcal{N}=1$ SCFTs at low-energies. They arise when a tree-level superpotential for $\Phi$ lifts the flat directions of $\mathcal{N}=2$ moduli space, leaving vacua that include mutually non-local monopoles as massless degrees of freedom. The conditions for a tree-level superpotential to lead to an $\mathcal{N}=1$ AD point include an $\mathcal{N}=2$ curve with triple or higher-order roots and a matrix model curve that vanishes at these roots. the $\mathcal{N}=2$ and matrix model curves via the solution of the factorization problem [13,5]:

$$
\begin{gathered}
y^{2}=P_{N}(x)^{2}-4 \Lambda^{2 N}=F_{2 n}(x) H_{N-n}(x)^{2} \\
y_{m}^{2}=W_{k}^{\prime}(x)^{2}+f_{k-1}(x)=F_{2 n}(x) Q_{k-n}(x)^{2} .
\end{gathered}
$$

For simplicity, we shall assume that the degree $k+1$ of the tree-level superpotential satisfies $k<N$, so that the polynomials $H_{N-n}$ and $Q_{k-n}$ are independent.

Notice that the $\mathcal{N}=2$ curve is comprised of two $N$ th-degree polynomial factors $P_{N}(x) \pm 2 \Lambda^{N}$ that cannot share any common roots. Thus the $\mathcal{N}=2$ curve can have at most an $N$ th order root, at which $P_{N}(x)=x^{N} \pm 2 \Lambda^{N}$. We will restrict our attention to the $\mathcal{N}=1$ singularities that can be obtained from these maximally singular points, as this will simplify our calculations considerably. At the same time, we do not expect to lose too much in the way of physics with such a restriction, since for a given $N$ the maximal $\mathrm{AD}$ points are the locations in moduli space where the largest number $(N-1)$ of mutually non-local, linearly independent monopoles become simultaneously massless. These $N-1$ monopoles are charged under $\left[\frac{N}{2}\right]$ of the $U(1)$ factors [22].

To understand the relationship between the $\mathcal{N}=1$ and the $\mathcal{N}=2 \mathrm{AD}$ points, it helps to study in greater detail the factorization problem (4.1). At the maximal AD point, $P_{N}(x)=x^{N}-2 \Lambda^{N}$ (we choose the minus sign without loss of generality) and the $\mathcal{N}=2$ curve takes the form

$$
y^{2}=x^{N}\left(x^{N}-4 \Lambda^{N}\right) .
$$

4 As noted in [19], this second condition is necessary in order for the non-local monopoles to remain uncondensed. A non-zero monopole condensate will produce a mass gap in the $U(1)$ under which it is charged. 
The $\mathcal{N}=2$ curve must factorize into the polynomials $F_{2 n}(x)$ and $H_{N-n}(x)^{2}$. Thus, for a given $N$ and $n$, we must have

$$
\begin{aligned}
& H_{N-n}(x)=x^{N-n} \\
& F_{2 n}(x)=x^{2 n-N}\left(x^{N}-4 \Lambda^{N}\right),
\end{aligned}
$$

implying the following form for the matrix model curve:

$$
y_{m}^{2}=F_{2 n}(x) Q_{k-n}(x)^{2}=\left(x^{n} Q_{k-n}(x)\right)^{2}-4 \Lambda^{N} x^{2 n-N} Q_{k-n}(x)^{2}
$$

Recall that we are assuming $k<N$; thus the second term is a polynomial of degree $<k$. Therefore we have

$$
\begin{aligned}
& W_{k}^{\prime}(x)=x^{n} Q_{k-n}(x) \\
& f_{k-1}(x)=-4 \Lambda^{N} x^{2 n-N} Q_{k-n}(x)^{2} .
\end{aligned}
$$

Together, (4.3) and (4.5) represent the complete solution to the factorization problem at the maximal AD point. We see that the maximal AD point actually leads to a number of continuous families of $\mathcal{N}=1$ vacua, obtained by varying the parameters of $Q_{k-n}(x)$. These continuous families are indexed by the integers $(k, n)$, which must satisfy the inequalities

$$
\left[\frac{N+1}{2}\right] \leq n \leq k<N .
$$

To avoid overcounting vacua, we will assume that $Q_{k-n}(x)$ is non-zero at $x=0$.

Not all of these $\mathcal{N}=1$ vacua will to lead to different interacting CFTs in the IR, however. Rather, we expect that many of the details of these vacua will become irrelevant in the IR, leading to a much smaller, discrete set of universality classes of $\mathcal{N}=1$ SCFTs. The simplest possibility is that the features of the IR theory are determined by the most relevant term of the tree-level superpotential. Let us write

$$
Q_{k-n}(x)=\sum_{i=0}^{k-n} q_{i} x^{i}
$$

with $q_{k-n}=1$ and $q_{0} \neq 0$. Then using (4.5) to relate $W(x)$ to $Q_{k-n}(x)$, we see that the most relevant (i.e. lowest-order) term in the tree-level superpotential is

$$
W(\Phi)=\frac{q_{0}}{n+1} \operatorname{Tr} \Phi^{n+1}+\mathcal{O}\left(\operatorname{Tr} \Phi^{n+2}\right) .
$$

As we flow down to the IR, we expect the operator $\operatorname{Tr} \Phi^{n+1}$ to become marginal or irrelevant, and the higher order terms in $W(\Phi)$ to become irrelevant. This suggests that 


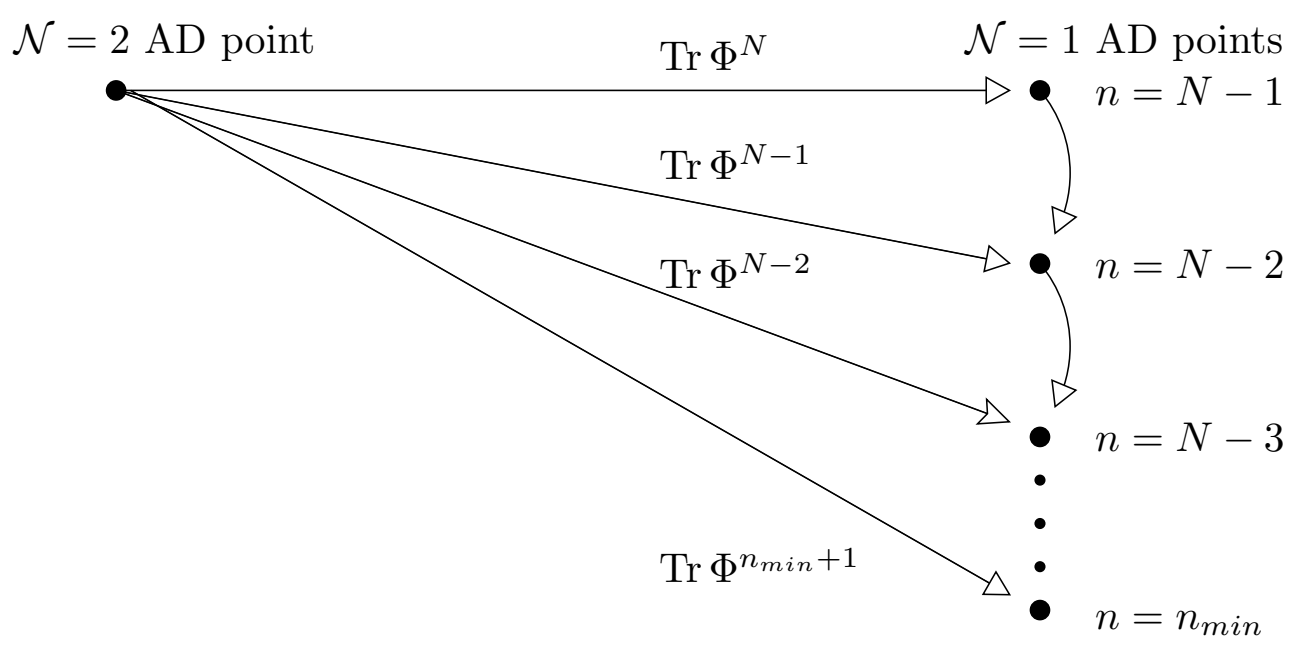

Figure 1: A schematic depiction of the proposed renormalization group flow between $A D$ points. As usual, the arrows indicate flow towards the IR. Starting from the $\mathcal{N}=2$ AD point (no tree-level superpotential), we can flow to a particular $\mathcal{N}=1 A D_{n}$ point by perturbing with the operator $\operatorname{Tr} \Phi^{n+1}$. Similarly, starting from an $A D_{n}$ point, we can flow to an $A D_{m}$ point with $m<n$ by perturbing with $\operatorname{Tr} \Phi^{m+1}$. The $R G$ cascade must terminate at $n=n_{\text {min }}$; perturbing further with relevant operators will send the theory to a trivial IR fixed point.

the IR physics depends only on the degree of singularity $n+1$ at $x=0$ of the tree-level superpotential, and not on its overall degree $k+1$. We are thus led to propose that the universality classes are labelled by $n$ alone. Let us call these universality classes $A D_{n}$ for want of a better name.

We can adduce some evidence for our proposal from the recent work of [19]. There, an attempt was made to define scaling operators and calculate their dimensions for the $\mathcal{N}=1 A D$ points obtained with monomial superpotential $W^{\prime}(x)=x^{n}$. The analysis was restricted to the range $n>n_{m i n}$, and it was found that the scaling dimensions of appropriately defined chiral operators depended only on the behavior of the matrix model curve very near $x=0$. From (4.4), we see that near $x=0$ the matrix model curve takes the form

$$
y_{m}^{2} \approx-4 \Lambda^{N} q_{0} x^{2 n-N}
$$

and in particular is independent of the overall degree $k$ of the superpotential. Thus, our proposal that the $\mathcal{N}=1 \mathrm{AD}$ points depend only on $n$, the degree of the most relevant term in the superpotential, and not on the overall degree $k$, is consistent with the approach of [19]. 
If our proposal is correct, we are led to a satisfying picture of the IR renormalization group flow as a cascade-like descent to CFTs of smaller and smaller $n$, as shown in figure 1. Starting from the $\mathcal{N}=2$ maximal AD point with $W(x)=0$ we can perturb by a monomial superpotential $W^{\prime}(x)=x^{n}$, inducing RG flow to the $\mathcal{N}=1 A D_{n}$ point. Perturbing further by a monomial term of lower degree $W^{\prime}(x) \rightarrow W^{\prime}(x)+x^{n-1}$ takes us to the $A D_{n-1}$ point. In this manner, we can descend from the $\mathcal{N}=2 \mathrm{AD}$ point down successively through the $\mathcal{N}=1 A D_{n}$ points via superpotential perturbations. The descent must end at the minimal value of $n_{\min }=\left[\frac{N+1}{2}\right]$; perturbing by a monomial of lower degree will send us to a trivial free theory in the IR.

There has been some debate as to whether the minimal value $n_{\min }$ corresponds to an interacting or a free IR theory. Although the authors of [19] restricted their analysis to $n>n_{\text {min }}$, they suggested that the IR theory was trivial and free for the borderline case $n=n_{\min }$. For $N$ even, this suggestion is almost certainly correct. As pointed out in [19], the monopole condensate corresponding to a given double root $p$ of the $\mathcal{N}=2$ curve is proportional to the matrix model curve at $p$ [27]:

$$
\langle q \tilde{q}\rangle \propto y_{m}(x=p)
$$

When $N$ is even, the matrix model curve is non-zero at $x=0$, and therefore the monopoles remain condensed even at the AD point. Thus the theory is indeed massive and trivial.

On the other hand, $y_{m}=0$ at $x=0$ for $N$ odd, and therefore the monopole condensates and the mass gap vanish at the $\mathrm{AD}$ point. Note that $N=3, n_{\min }=2$ is the original $\mathcal{N}=1 \mathrm{AD}$ point discussed in the work of Argyres and Douglas. The vanishing of the monopole condensates for the original $\mathcal{N}=1 A D$ point was also demonstrated explicitly in [28]. But the authors of [19] argued that the IR theory for $n=n_{\min }$ and $N$ odd is nevertheless trivial, because the matrix model curve is non-singular at $x=0$. While this fact does not necessarily signify anything per se, it does imply that the chiral operators defined in [19] do not exhibit scaling behavior. Thus there is no evidence for an interacting IR theory in that approach. However we wish to argue in the rest of this section that there does in fact exist evidence for an interacting theory when $N$ is odd. By studying both the effective superpotential in both the glueball and the strong-coupling descriptions, we will show that the effective descriptions look dramatically different for $N$ even and $N$ odd. We will confirm that IR theory is indeed trivial for $N$ even, but seems to be non-trivial for $N$ odd. 


\subsection{The effective glueball superpotential}

We start with the case of $N$ odd. The matrix model curve for $N$ odd and $n=n_{\min }$ is

$$
y_{m}^{2}=x\left(x^{N}-4 \Lambda^{N}\right)+g_{n-1}(x),
$$

where we are using $g_{n-1}(x)$ to denote the off-shell fluctuations of the matrix model curve. The $n$ coefficients of $g_{n-1}(x)$ are related to the glueball fields through the periods of $y_{m}$. Notice that we have assumed $k=n$, i.e. a monomial superpotential. We will continue to assume this for the rest of the section. If the IR physics is determined by $n$ alone, as we have proposed, then we lose no generality in making this simplifying assumption.

We wish to compute the effective glueball superpotential $W_{\text {eff }}$ derived from this matrix model curve. While we will not be able to obtain an explicit formula for $W_{\text {eff }}$ for general $N$, we will be able to constrain the form of $W_{\text {eff }}$ using a discrete $\mathbf{Z}_{N}$ symmetry. This alone will allow some surprising conclusions to be drawn about the low-energy theory. The $\mathbf{Z}_{N}$ symmetry is closely related to the $U(1)_{R}$ symmetry of the microscopic theory. Let us now take a moment to describe in detail how the $\mathbf{Z}_{N}$ symmetry comes about. Here we will give only heuristic arguments for the existence of such a symmetry; we refer the reader to appendix B for a more detailed derivation using matrix model techniques.

The microscopic theory has a $U(1)_{R}$ symmetry which is unbroken by the monomial tree-level superpotential $W(\Phi)=\operatorname{Tr} \Phi^{n+1}$, provided we give $\Phi$ an $R$-charge $\frac{2}{n+1}$. Quantum mechanically, $P_{N}(x)=x^{N}-2 \Lambda^{N}$ at the AD point, and thus $\left\langle\operatorname{Tr} \Phi^{j}\right\rangle=0$ for $j=1, \ldots, N-1$ and $\left\langle\operatorname{Tr} \Phi^{N}\right\rangle=2 \Lambda^{N}$. As a result, the $U(1)_{R}$ symmetry is spontaneously broken down to $\mathbf{Z}_{N}$ by the expectation value of $\operatorname{Tr} \Phi^{N}$. Under the residual $\mathbf{Z}_{N}$ symmetry, $\Phi$ and the superpotential can be chosen to transform as

$$
\Phi \rightarrow e^{\frac{4 \pi i}{N}} \Phi, \quad W(\Phi) \rightarrow e^{\frac{6 \pi i}{N}} W(\Phi) .
$$

Now consider integrating out $\Phi$ and passing to the low-energy effective description in terms of the glueball fields. Since the residual $\mathbf{Z}_{N}$ symmetry is unbroken in the exact quantum theory, the effective superpotential should also transform as

$$
W_{e f f} \rightarrow e^{\frac{6 \pi i}{N}} W_{e f f}
$$

under the action of $\mathbf{Z}_{N}$. (This can be shown more rigorously through explicit matrix model computations; see appendix B.) 
It remains to determine how the glueball fields of the low-energy theory, or equivalently the coefficients of $g_{n-1}(x)$, transform under $\mathbf{Z}_{N}$. But this is not hard to see. The on-shell matrix model curve is $y_{m}^{2}=x\left(x^{N}-4 \Lambda^{N}\right)$; if we make the natural identification of the $R$-charge of $x$ with the $R$-charge of $\Phi$, it follows that $y_{m}^{2}$ has charge $\frac{4 \pi}{N}$ under $\mathbf{Z}_{N}$. This will also be true off-shell if the coefficients of $g_{n-1}(x)=\sum_{j=0}^{n-1} g_{j} x^{j}$ transform as

$$
g_{j} \rightarrow e^{4 \pi i(1-j) / N} g_{j} .
$$

Together, (4.13) and (4.14) describe the action of the $\mathbf{Z}_{N}$ symmetry on the low-energy theory. We are now ready to understand the implications of this discrete symmetry on the effective superpotential. Recalling that $W_{\text {eff }}$ must be holomorphic in the fields $g_{j}$, we find that the $\mathbf{Z}_{N}$ symmetry severely restricts the form of the effective superpotential:

$$
W_{\text {eff }}=g_{n-1} G\left(g_{0}^{N}, g_{1}, g_{2} g_{0}, \ldots, g_{n-1} g_{0}^{n-2}\right)
$$

for some holomorphic function $G$. While this does not determine the full form of $W_{\text {eff }}$, it does allow us to write down the linear and quadratic terms up to undetermined constants:

$$
W_{e f f} \approx c_{0} g_{n-1}+\sum_{i=1}^{\left[\frac{n}{2}\right]} c_{i} g_{i} g_{n-i}+\ldots
$$

The linear term can actually be eliminated, because if $c_{0} \neq 0$, this merely tells us that $\left\langle g_{1}\right\rangle \approx-c_{0} / c_{1} \neq 0$. But from the form of the matrix model curve (4.11), we deduce that giving a vev to $g_{1}$ is indistinguishable from redefining $\Lambda$ by a constant. Therefore we might as well redefine $\Lambda$ so that $c_{1}=0$.

No other terms are possible at quadratic order in the fields. In particular, a mass term for $g_{0}$ is forbidden by the $\mathbf{Z}_{N}$ symmetry! Mass terms are clearly allowed for the other $n-1$ fields. Therefore, in the absence of additional, accidental symmetries (and assuming regularity of the Kähler potential) we except there to be exactly one massless glueball in the low-energy spectrum for $N$ odd and $n=n_{\text {min }}$.

Now let us consider the case of $N$ even. The matrix model curve for $N$ even and $n=n_{\min }$ is

$$
y_{m}^{2}=\left(x^{N}-4 \Lambda^{N}\right)+g_{n-1}(x) .
$$

The arguments for the $\mathbf{Z}_{N}$ symmetry are nearly identical to those given above for $N$ odd. Therefore we save the details for the appendix and merely summarize the results here. We once again have $x \rightarrow e^{\frac{4 \pi i}{N}} x$, but now

$$
W_{e f f} \rightarrow e^{\frac{4 \pi i}{N}} W_{e f f}
$$


and

$$
g_{j} \rightarrow e^{-\frac{4 \pi i j}{N}} g_{j}
$$

This restricts the form of the effective superpotential to be

$$
W_{e f f}=g_{n-1} F\left(g_{0}, g_{1} g_{n-1}, \ldots, g_{n-2} g_{n-1}^{n-2}, g_{n-1}^{n}\right)
$$

for some holomorphic function $F$. To quadratic order, the possible terms are therefore

$$
W_{e f f} \approx c_{0} g_{n-1}+\sum_{i=0}^{\left[\frac{n-1}{2}\right]} c_{i} g_{i} g_{n-1-i}
$$

Again the linear term can be eliminated by a shift in $\Lambda$. However, in contrast to the odd $N$ case, no mass terms are forbidden for the glueball fields for even $N$. In the absence of additional symmetries, we expect all $c_{i} \neq 0$. This is evidence that for $N$ even and $n=n_{\min }$ all the fluctuations are massive around the AD point. This is quite different from the case of $N$ odd!

\subsection{The strong coupling superpotential}

In this subsection, we will analyze the effective superpotential in the strong-coupling description, where we treat the tree-level superpotential as a small perturbation of the underlying $\mathcal{N}=2$ theory. In the strong coupling analysis, the low-energy degrees of freedom are the fields $u_{r}=\frac{1}{r} \operatorname{Tr} \Phi^{r}$ and some number of light monopole fields $q_{l}, \tilde{q}_{l}$, $l=1, \ldots, N-n$. Their interactions are described by the effective superpotential

$$
W_{e f f}=W(u)+\sum_{l=1}^{N-n} M_{l}(u) q_{l} \tilde{q}_{l},
$$

where $W(u)$ is the tree-level superpotential and $M_{l}(u)$ are the monopole masses [14]. Integrating out the monopole fields implies $M_{l}(u)=0$ and leads to an effective superpotential for the fields $u_{r}, r=1, \ldots, n$ alone. For the monomial superpotential under consideration, the effective superpotential after integrating out the monopole fields is simply

$$
W_{e f f}=u_{n+1}\left(u_{1}, \ldots, u_{n}\right)
$$

where the $N-n$ equations $M_{l}(u)=0$ are used to re-express $u_{n+j}$ in terms of the fields $u_{1}, \ldots, u_{n}$. Determining the function $u_{n+1}\left(u_{1}, \ldots, u_{n}\right)$ will be the focus of the remainder of our strong-coupling analysis. 
In practice, it is very difficult to determine the function $u_{n+1}\left(u_{1}, \ldots, u_{n}\right)$ directly from the massless monopole equations. Fortunately, there exists an easier way, namely the factorization of the $\mathcal{N}=2$ curve. Requiring there to be $N-n$ massless monopoles is equivalent to requiring the $\mathcal{N}=2$ curve to factorize into $N-n$ double roots [18]:

$$
y^{2}=P_{N}(x)^{2}-4 \Lambda^{2 N}=F_{2 n}(x) H_{N-n}(x)^{2} .
$$

If we write

$$
P_{N}(x)=\sum_{k=0}^{N} s_{k} x^{N-k}
$$

with $s_{0}=1$, then the coefficients of $P_{N}(x)$ are simply related to the fields $u_{r}$ through the equations

$$
k s_{k}+\sum_{r=1}^{k} r u_{r} s_{k-r} .
$$

The factorization (4.24) amounts to a system of $2 N$ equations for $2 N+n$ unknowns (the coefficients of $P_{N}, F_{2 n}$ and $H_{N-n}$ ), and thus only $n$ are independent. Using (4.26), we can express all of the coefficients in terms of $u_{1}, \ldots, u_{n}$, and this will produce the desired dependence of $u_{n+j}$ on $u_{1}, \ldots, u_{n}$.

We can simplify the problem even further in the case of the maximal AD point. At the maximal AD point, $\left.P_{N}(x)\right|_{A D}=x^{N}-2 \Lambda^{N}$ and

$$
\left.y^{2}\right|_{A D}=\left.\left(P_{N}(x)+2 \Lambda^{N}\right)\left(P_{N}(x)-2 \Lambda^{N}\right)\right|_{A D}=x^{N}\left(x^{N}-4 \Lambda^{N}\right) .
$$

The roots in the second factor of (4.27) are all separated by $\mathcal{O}(\Lambda)$, so perturbing $\left\langle u_{i}\right\rangle_{A D} \rightarrow$ $\left\langle u_{i}\right\rangle_{A D}+\delta u_{i}$ slightly away from the AD point on a branch with $N-n$ double roots must leave all the double roots in the first factor. Therefore we have reduced the factorization problem from a system of $2 N$ equations to a system of $N$ equations:

$$
P_{N}(x)+2 \Lambda^{N}= \begin{cases}H_{N-n}(x)^{2} & N \text { even } \\ (x-a) H_{N-n}(x)^{2} & N \text { odd }\end{cases}
$$

For $N$ even, the factorization problem can be neatly solved as a power series in $u_{1}, \ldots, u_{n}$. The resulting effective superpotential is given to quadratic order by

$$
W_{\text {eff }}=\frac{1}{4} \sum_{r=1}^{n} u_{r} u_{n+1-r}+\mathcal{O}\left(u^{3}\right) \text {. }
$$


Thus the strong-coupling effective superpotential (4.29) for $N$ even is regular at the AD point, and all the fluctuations about the vacuum are massive.

We have skipped the full proof of (4.29), because while straightforward, it is messy and not very illuminating. But perhaps it will help to illustrate the general idea by working out the simplest example of $N=4$. In this case, $n=2$, so we are interested in finding $u_{3}\left(u_{1}, u_{2}\right)$ by solving (4.28):

$$
P_{4}(x)+2 \Lambda^{4}=H_{2}(x)^{2}=\left(x^{2}+c_{1} x+c_{2}\right)^{2} .
$$

Substituting (4.25) for $P_{4}(x)$ and equating the coefficients of the two sides of (4.30) immediately leads to

$$
c_{1}=\frac{1}{2} s_{1}, \quad c_{2}=\frac{1}{2}\left(s_{2}-\frac{1}{4} s_{1}^{2}\right)
$$

and so

$$
s_{3}=2 c_{1} c_{2}=\frac{1}{2} s_{1}\left(s_{2}-\frac{1}{4} s_{1}^{2}\right) .
$$

It is simple enough now to use (4.26) to translate (4.32) into a formula for $u_{3}$. The result is

$$
u_{3}=\frac{1}{2} u_{1} u_{2}-\frac{1}{24} u_{1}^{3}
$$

which verifies the general result (4.29) for $N=4$.

Unfortunately the story is not so simple for $N$ odd. In general, $u_{n+1}$ will be determined from an $n(n+1)$ degree quasi-homogeneous polynomial equation

$$
R_{n(n+1)}\left(u_{1}, \ldots, u_{n+1}\right)=0 .
$$

Thus the complexity of the solution grows rapidly with increasing $N$. One can simplify things somewhat by restricting the analysis to $S U(N)$; this amounts to setting $u_{1}=0$. The trace can always be restored at the end of the calculation by translating the $S U(N)$ variables back into the $U(N)$ variables. Even with this simplification, however, we are not able to obtain a general formula for the polynomial $R_{n(n+1)}$. To illustrate the rapidly growing complexity of these polynomials, we display here the results for $S U(3)$ and $S U(5)$ :

$$
\begin{aligned}
& R_{6}\left(u_{2}, u_{3}\right)=27 u_{3}^{2}-4 u_{2}^{3} \\
& R_{12}\left(u_{2}, u_{3}, u_{4}\right)=1600 u_{4}^{3}-1360 u_{2}^{2} u_{4}^{2}+\left(384 u_{2}^{4}-360 u_{2} u_{3}^{2}\right) u_{4}-\left(36 u_{2}^{6}-92 u_{2}^{3} u_{3}^{2}+135 u_{3}^{4}\right) .
\end{aligned}
$$


Although for $N$ odd we lack an explicit formula for $W_{\text {eff }}$, we can make a few general statements. Because of the presence of higher powers of $u_{n+1}$, the solution $u_{n+1}\left(u_{1}, \ldots, u_{n}\right)$ of $R_{n(n+1)}=0$ will in general be non-analytic at $u_{1}=\ldots=u_{n}=0$. In particular, the second derivatives of $W_{\text {eff }}$ will generally be divergent at the maximal AD point. For instance, the effective superpotential for $S U(3)$ is

$$
W_{e f f}=u_{3}\left(u_{2}\right)=\sqrt{\frac{4}{27}} u_{2}^{3 / 2},
$$

and the field $u_{2}$ is seen to have a divergent mass term at $u_{2}=0$. (The formula for $U(3)$ will be somewhat more complicated, although the conclusions are unchanged.) Thus for $N$ odd, the strong-coupling analysis produces rather pathological singularities in the effective superpotential. Such pathologies in the effective superpotential generally indicate a breakdown of the effective description and signal the presence of extra massless fields. Contrast this with the case of $N$ even, where both the glueball analysis and the strong-coupling analysis resulted in perfectly regular superpotentials with all fluctuations massive. This suggests that the additional massless fields are precisely the mutually non-local monopole fields that remain uncondensed at the AD point for $N$ odd. We take this to be evidence that the $\mathcal{N}=1 \mathrm{AD}$ point with $n=n_{\min }$ and $N$ odd is indeed a non-trivial, interacting CFT at low-energies.

\section{Acknowledgements}

First and foremost I would like to thank my advisor N. Seiberg for suggesting this problem and for his constant guidance and support throughout every stage of this work. It is also a pleasure to thank F. Cachazo, S. Murthy, M. Douglas, K. Intriligator and M. Strassler for useful discussions. This work was supported by an NSF Graduate Research Fellowship. Any opinions, findings, and conclusions or recommendations expressed in this material are those of the author and do not necessarily reflect the views of the National Science Foundation.

\section{Appendix A. Calculating derivatives of periods on the $n=2$ branch}

In this appendix, we evaluate the derivatives of the periods $S_{i}$ and $\Pi_{i}$ of the matrix model curve on the $n=2$ branch, with cubic tree-level superpotential. The periods of 
interest are the glueball fields $S_{i}$ :

$$
\begin{aligned}
& 2 \pi i S_{1}=\int_{r_{1}}^{r_{2}} \sqrt{\left(x-r_{1}\right)\left(x-r_{2}\right)\left(x-r_{3}\right)\left(x-r_{4}\right)} d x \\
& 2 \pi i S_{2}=\int_{r_{3}}^{r_{4}} \sqrt{\left(x-r_{1}\right)\left(x-r_{2}\right)\left(x-r_{3}\right)\left(x-r_{4}\right)} d x
\end{aligned}
$$

and their conjugate periods:

$$
\begin{aligned}
& 2 \pi i \Pi_{1}=\int_{-\Lambda_{0}}^{r_{1}} \sqrt{\left(x-r_{1}\right)\left(x-r_{2}\right)\left(x-r_{3}\right)\left(x-r_{4}\right)} d x \\
& 2 \pi i \Pi_{2}=\int_{\Lambda_{0}}^{r_{4}} \sqrt{\left(x-r_{1}\right)\left(x-r_{2}\right)\left(x-r_{3}\right)\left(x-r_{4}\right)} d x .
\end{aligned}
$$

These periods are combined to give the effective glueball superpotential:

$$
W_{e f f}=2 \pi i\left(N_{1} \Pi_{1}+N_{2} \Pi_{2}+b_{2} S_{2}\right)+2 N S \log \left(\frac{\Lambda}{\Lambda_{0}}\right) .
$$

Let us start with the derivatives of $\Pi_{i}$ :

$$
\begin{aligned}
& 4 \pi i \frac{\partial \Pi_{1}}{\partial f_{j}}=\int_{-\Lambda_{0}}^{r_{1}} \frac{x^{j}}{\sqrt{\left(x-r_{1}\right)\left(x-r_{2}\right)\left(x-r_{3}\right)\left(x-r_{4}\right)}} d x \\
& 4 \pi i \frac{\partial \Pi_{2}}{\partial f_{j}}=-\int_{r_{4}}^{\Lambda_{0}} \frac{x^{j}}{\sqrt{\left(x-r_{1}\right)\left(x-r_{2}\right)\left(x-r_{3}\right)\left(x-r_{4}\right)}} d x .
\end{aligned}
$$

With some effort, these integrals can be evaluated and expressed in terms of elliptic integrals of the first and third kinds:

$$
\begin{aligned}
& 4 \pi i \frac{\partial \Pi_{1}}{\partial f_{0}}=\frac{2}{\sqrt{\left(r_{4}-r_{2}\right)\left(r_{3}-r_{1}\right)}} F(\theta \mid R) \\
& 4 \pi i \frac{\partial \Pi_{1}}{\partial f_{1}}=\log \left(\frac{r_{4}+r_{3}-r_{2}-r_{1}}{4 \Lambda_{0}}\right)+\frac{2\left(r_{1} F(\theta \mid R)+\left(r_{2}-r_{1}\right) \Pi(n ; \theta \mid R)\right)}{\sqrt{\left(r_{4}-r_{2}\right)\left(r_{3}-r_{1}\right)}} \\
& 4 \pi i \frac{\partial \Pi_{2}}{\partial f_{0}}=\frac{2}{\sqrt{\left(r_{4}-r_{2}\right)\left(r_{3}-r_{1}\right)}} \Delta F_{2}\left(r_{i}\right) \\
& 4 \pi i \frac{\partial \Pi_{2}}{\partial f_{1}}=\log \left(\frac{r_{4}+r_{3}-r_{2}-r_{1}}{4 \Lambda_{0}}\right)+\frac{2\left(r_{1} \Delta F_{2}\left(r_{i}\right)+\left(r_{2}-r_{1}\right) \Delta \Pi_{2}\left(r_{i}\right)\right)}{\sqrt{\left(r_{4}-r_{2}\right)\left(r_{3}-r_{1}\right)}}
\end{aligned}
$$

where

$$
\begin{aligned}
& \Delta F_{2}\left(r_{i}\right)=F(\theta \mid R)-F\left(\frac{\pi}{2} \mid R\right) \\
& \Delta \Pi_{2}\left(r_{i}\right)=\Pi(n ; \theta \mid R)-\Pi\left(n ; \frac{\pi}{2} \mid R\right) \\
& \sin ^{2} \theta=\frac{r_{4}-r_{2}}{r_{4}-r_{1}}, \quad R=\frac{\left(r_{3}-r_{2}\right)\left(r_{4}-r_{1}\right)}{\left(r_{3}-r_{1}\right)\left(r_{4}-r_{2}\right)}, \quad n=\frac{r_{3}-r_{2}}{r_{3}-r_{1}} .
\end{aligned}
$$


The formulas in (A.5) are exact, up to terms of $\mathcal{O}\left(1 / \Lambda_{0}\right)$ which are irrelevant in the $\Lambda_{0} \rightarrow \infty$ limit. Here $F$ and $\Pi$ are the elliptic integrals of the first and third kinds, respectively. We recall their definitions:

$$
\begin{aligned}
& F(\theta \mid R)=\int_{0}^{\theta} \frac{d \theta^{\prime}}{\sqrt{1-R \sin ^{2} \theta^{\prime}}} \\
& \Pi(n ; \theta \mid R)=\int_{0}^{\theta} \frac{d \theta^{\prime}}{\left(1-n \sin ^{2} \theta^{\prime}\right) \sqrt{1-R \sin ^{2} \theta^{\prime}}} .
\end{aligned}
$$

Using (A.5) in the formula (A.3) for the effective glueball superpotential immediately results in the glueball equations of motion (3.2) (for $b_{2}=0$ ).

Now let us turn to the derivatives of the $S_{i}$. It actually suffices to consider only the derivatives of $S_{2}$, since $S_{1}+S_{2}=S=-\frac{1}{4} f_{1}$ :

$$
\begin{aligned}
& 4 \pi i \frac{\partial S_{2}}{\partial f_{0}}=-i \int_{r_{3}}^{r_{4}} \frac{1}{\sqrt{\left(x-r_{1}\right)\left(x-r_{2}\right)\left(x-r_{3}\right)\left(r_{4}-x\right)}} d x \\
& 4 \pi i \frac{\partial S_{2}}{\partial f_{1}}=-i \int_{r_{3}}^{r_{4}} \frac{x}{\sqrt{\left(x-r_{1}\right)\left(x-r_{2}\right)\left(x-r_{3}\right)\left(r_{4}-x\right)}} d x .
\end{aligned}
$$

Note that we have implicitly chosen the branch of the square root in the denominator by pulling out a factor of $-i$ in front of the integral. One can verify that this is the correct branch of the square root to choose by computing $S_{1}$ and making sure that they indeed combine to give $-\frac{1}{4} f_{1}$. The integrals (A.8) evaluate to:

$$
\begin{aligned}
& 4 \pi i \frac{\partial S_{2}}{\partial f_{0}}=-\frac{2 i}{\sqrt{\left(r_{4}-r_{2}\right)\left(r_{3}-r_{1}\right)}} F\left(\frac{\pi}{2} \mid R_{2}\right) \\
& 4 \pi i \frac{\partial S_{2}}{\partial f_{1}}=-\frac{2 i}{\sqrt{\left(r_{4}-r_{2}\right)\left(r_{3}-r_{1}\right)}}\left(r_{2} F\left(\frac{\pi}{2} \mid R_{2}\right)+\left(r_{3}-r_{2}\right) \Pi\left(n_{2} ; \frac{\pi}{2} \mid R_{2}\right)\right)
\end{aligned}
$$

where $R_{2}$ and $n_{2}$ are given by

$$
R_{2}=1-R=\frac{\left(r_{2}-r_{1}\right)\left(r_{4}-r_{3}\right)}{\left(r_{4}-r_{2}\right)\left(r_{3}-r_{1}\right)}, \quad n_{2}=\frac{1-R}{1-n}=\frac{r_{4}-r_{3}}{r_{4}-r_{2}} .
$$

Finally, we will compute the partial derivative $\frac{\partial \Pi_{2}}{\partial S_{1}}$ near the $n=1 / n=2$ singularity. This calculation is relevant for obtaining the matrix of coupling constants at the singularity. First, we use the chain rule to relate the derivative with respect to $S_{1}$ to derivatives with respect to $f_{0}$ and $f_{1}$ :

$$
\begin{aligned}
\frac{\partial \Pi_{2}}{\partial S_{1}} & =\frac{\partial \Pi_{2}}{\partial f_{1}} \frac{\partial f_{1}}{\partial S_{1}}+\frac{\partial \Pi_{2}}{\partial f_{0}} \frac{\partial f_{0}}{\partial S_{1}} \\
& =4\left(\kappa \frac{\partial \Pi_{2}}{\partial f_{0}}-\frac{\partial \Pi_{2}}{\partial f_{1}}\right)
\end{aligned}
$$


Here we have defined the ratio $\kappa=\frac{\partial S_{2}}{\partial f_{1}} / \frac{\partial S_{2}}{\partial f_{0}}$, and we have used the fact that $S_{1}=S-S_{2}=$ $-4 f_{1}-S_{2}$ to eliminate $S_{1}$. The ratio $\kappa$ is the source of the logarithmic singularity. Using (A.9) gives:

$$
\kappa=r_{2}+\frac{\left(r_{3}-r_{2}\right) \Pi\left(n_{2} ; \frac{\pi}{2} \mid R_{2}\right)}{F\left(\frac{\pi}{2} \mid R_{2}\right)} .
$$

As $r_{2}-r_{3} \rightarrow 0$, we see from (A.10) that $n_{2}$ and $R_{2} \rightarrow 1$. The limiting behavior of $F\left(\frac{\pi}{2} \mid R_{2}\right)$ as $R_{2} \rightarrow 1$ is

$$
F\left(\frac{\pi}{2} \mid R_{2}\right)=\frac{1}{2} \log \left(\frac{16}{1-R_{2}}\right)+\mathcal{O}\left(1-R_{2}\right)=\frac{1}{2} \log \left(\frac{16}{R}\right)+\mathcal{O}(R) .
$$

On the other hand, the limiting behavior of $\left(r_{3}-r_{2}\right) \Pi\left(n_{2} ; \frac{\pi}{2} \mid R_{2}\right)$ is finite, as one can see by returning to the integral definition (A.7) of $\Pi$. Let us write $1 / n_{2}=1+\epsilon$ and $1 / R_{2}=1+\alpha \epsilon$, where $\epsilon \rightarrow 0$ and $\alpha$ is some constant. Then from (A.7), we obtain

$$
\begin{aligned}
\Pi\left(n_{2} ; \frac{\pi}{2} \mid R_{2}\right) & =\frac{1}{n_{2} \sqrt{R_{2}}} \int_{0}^{1} \frac{d t}{\left(t^{2}+\epsilon\right) \sqrt{\left(t^{2}+\alpha \epsilon\right)\left(1-t^{2}\right)}} \\
& =\frac{1}{\epsilon n_{2} \sqrt{R_{2}}} \int_{0}^{\frac{1}{\sqrt{\epsilon}}} \frac{d t}{\left(t^{2}+1\right) \sqrt{\left(t^{2}+\alpha\right)\left(1-\epsilon t^{2}\right)}} \\
& =\frac{r_{4}-r_{2}}{r_{3}-r_{2}} \sqrt{\frac{r_{3}-r_{1}}{r_{4}-r_{3}}} \theta+\mathcal{O}\left(\epsilon^{0}\right)
\end{aligned}
$$

where $\theta$ is defined in (3.3). Combining (A.13) and (A.14), we see that

$$
\kappa=r_{2}+\frac{2\left(r_{4}-r_{2}\right) \sqrt{\frac{r_{3}-r_{1}}{r_{4}-r_{3}}} \theta}{\log \left(\frac{16}{R}\right)}+\mathcal{O}(R) .
$$

Finally, we use the equations of motion (3.4) to simplify the formula (A.5) for the derivatives of $\Pi_{i}$, since we will only be interested in the matrix of coupling constants on-shell.

$$
\begin{aligned}
& 2 \pi i \frac{\partial \Pi_{2}}{\partial f_{0}}=-\frac{N_{1}}{N \sqrt{\left(r_{4}-r_{2}\right)\left(r_{3}-r_{1}\right)}} F\left(\frac{\pi}{2} \mid R\right) \\
& 2 \pi i \frac{\partial \Pi_{2}}{\partial f_{1}}=-\frac{N_{1}}{N \sqrt{\left(r_{4}-r_{2}\right)\left(r_{3}-r_{1}\right)}}\left(r_{1} F\left(\frac{\pi}{2} \mid R\right)+\left(r_{2}-r_{1}\right) \Pi\left(n ; \frac{\pi}{2} \mid R\right)\right) .
\end{aligned}
$$

As we approach the singularity, we can use (3.6) to replace $\theta \rightarrow \frac{\pi}{2} \frac{N_{2}}{N}$. Moreover $F\left(\frac{\pi}{2} \mid R\right)$ and $\Pi\left(n ; \frac{\pi}{2} \mid R\right) \rightarrow \frac{\pi}{2}$ as $R \rightarrow 0$. Using these facts and (A.16) and (A.15) in (A.11), we obtain at last our formula for $\frac{\partial \Pi_{2}}{\partial S_{1}}$ :

$$
\frac{\partial \Pi_{2}}{\partial S_{1}}=\frac{\pi i N_{1} N_{2}}{N^{2}} \frac{1}{\log \left(\frac{16}{R}\right)}+\mathcal{O}(R) .
$$




\section{Appendix B. The $\mathbf{Z}_{N}$ symmetry of the $\mathcal{N}=1$ AD point}

In section 4.1, we gave a heuristic derivation of the $\mathbf{Z}_{N}$ symmetry of the $\mathcal{N}=1$, $n=n_{\min } \mathrm{AD}$ points by using the microscopic $U(1)_{R}$ symmetry. In this appendix, we will derive the $\mathbf{Z}_{N}$ symmetry directly from the matrix model calculation of the glueball superpotential. As before, we must consider separately the cases of $N$ even and $N$ odd. We first consider the case of $N$ odd.

To begin, notice that for $N$ odd, the branch points of $y_{m}$ are located at $x_{0}=0$ and at the $N$ th roots of unity $x_{j}=e^{2 \pi i(j-1) / N}$ (we temporarily set $4 \Lambda^{N}=1$ for convenience). Let us define the $A$-cycles to be $A_{1}=(0,1)$ and $A_{i}=\left(x_{2 i-2}, x_{2 i-1}\right)$. Then the branch points and the $A$-cycles possess a natural $\mathbf{Z}_{N}$ symmetry, which we will take to be generated by $x \rightarrow e^{4 \pi i / N} x$. The matrix model curve (4.11) transforms as $y_{m}^{2} \rightarrow e^{4 \pi i / N} y_{m}^{2}$ under this transformation if we take the coefficients of $g_{n-1}(x)$ to transform with charges

$$
g_{j} \rightarrow e^{4 \pi i(1-j) / N} g_{j}
$$

Using the $\mathbf{Z}_{N}$ symmetry and various contour integrals, one can show that the periods of the one-form $T(x)$ are $N_{1}=1$ and $N_{i}=2, b_{i}=1$ for $i>1$. Thus the effective superpotential takes the following form for $N$ odd:

$$
W_{e f f}=\Pi_{1}+\sum_{i=2}^{n}\left(2 \Pi_{i}+S_{i}\right)
$$

We have dropped the usual term proportional to the bare gauge coupling, as this merely serves to renormalize $\Lambda_{0} \rightarrow \Lambda$.

In order to realize the $\mathbf{Z}_{N}$ symmetry explicitly in the effective superpotential, it will be useful to imagine deforming each cut $A_{i}$ so that it nearly touches the origin (recall that $A_{i}$ runs between adjacent $N$ th roots of unity). Then, at least for the purposes of computing the periods $\Pi_{i}$, we can consider the $x$ plane as consisting of $N$ "cuts" that run from $x=0$ to the $N$ roots of unity. Define the contours $\tilde{B}_{j}, j=1, \ldots, N$ which connect $\Lambda_{0}$ on the two sheets and run through the $j$ th "cut", and define the periods $\tilde{\Pi}_{j}$ of $R(x)$ based on these contours:

$$
\tilde{\Pi}_{j}=-\frac{1}{2} \oint_{\tilde{B}_{j}} \sqrt{x\left(x^{N}-1\right)+g_{n-1}(x)} d x .
$$


By various contour deformations, one can relate the original periods $\Pi_{i}$ to the $\tilde{\Pi}_{j}$, and one can show that the effective superpotential (B.2) simplifies to the following suggestive form:

$$
W_{e f f}=\sum_{j=1}^{N} \tilde{\Pi}_{j} .
$$

From the definition $(\mathbb{B . 3})$, it is clear that the $\mathbf{Z}_{N}$ transformation acts on the periods $\tilde{\Pi}_{j}$ as

$$
\tilde{\Pi}_{j}(g) \rightarrow e^{6 \pi i / N} \times \tilde{\Pi}_{j+2}(g) .
$$

It follows that $W_{\text {eff }}$ transforms with definite charge under the $\mathbf{Z}_{N}$ symmetry:

$$
W_{e f f} \rightarrow e^{6 \pi i / N} W_{e f f}
$$

This confirms the heuristic derivation of the $\mathbf{Z}_{N}$ symmetry given in section 4.1 for $N$ odd.

The calculation is very similar for $N$ even, so we omit most of the intermediate steps. The $N$ branch points of the matrix model curve are located at the $N$ th roots of unity $x_{j}=e^{2 \pi i(j-1) / N}$. We define the $A$-cycles to be $A_{i}=\left(x_{2 i-1}, x_{2 i}\right)$. Then $\mathbf{Z}_{N}$ symmetry again acts as $x \rightarrow e^{4 \pi i / N} x$, but this time the matrix model curve (4.17) is invariant, as long as we transform the coefficients of $g_{n-1}(x)$ with charges

$$
g_{j} \rightarrow e^{-4 \pi i j / N} g_{j}
$$

The periods of $T(x)$ are $N_{i}=2$ and $b_{i}=0$, so the effective superpotential takes the form

$$
W_{e f f}=2 \sum_{i=1}^{n} \Pi_{i}
$$

where again we omit the bare-coupling term. Once again, we consider deforming the cuts so that they nearly touch the origin, and we define the periods $\tilde{\Pi}_{i}, i=1, \ldots, N$. Similar arguments as for $N$ odd lead to the following expression for $W_{\text {eff }}$ :

$$
W_{e f f}=\sum_{j=1}^{N} \tilde{\Pi}_{j}-S .
$$

Now the $\mathbf{Z}_{N}$ transformation acts on $\tilde{\Pi}_{j}$ as

$$
\tilde{\Pi}_{j}(g) \rightarrow e^{4 \pi i / N} \times \tilde{\Pi}_{j+2}(g) .
$$

Moreover, since $S=-\frac{1}{4} g_{n-1}, S$ also transforms as $S \rightarrow e^{4 \pi i / N} S$ under the action of $\mathbf{Z}_{N}$. Therefore $W_{\text {eff }}$ again transforms with definite charge under the $\mathbf{Z}_{N}$ symmetry:

$$
W_{e f f} \rightarrow e^{4 \pi i / N} W_{e f f}
$$

as expected from the arguments of section 4.1 . 


\section{References}

[1] R. Dijkgraaf and C. Vafa, "Matrix models, topological strings, and supersymmetric gauge theories," Nucl. Phys. B 644, 3 (2002) arXiv:hep-th/0206255.

[2] R. Dijkgraaf and C. Vafa, "On geometry and matrix models," Nucl. Phys. B 644, 21 (2002) arXiv:hep-th/0207106.

[3] R. Dijkgraaf and C. Vafa, "A perturbative window into non-perturbative physics," arXiv:hep-th/0208048.

[4] F. Cachazo, M. R. Douglas, N. Seiberg and E. Witten, "Chiral rings and anomalies in supersymmetric gauge theory," JHEP 0212, 071 (2002) arXiv:hep-th/0211170.

[5] F. Cachazo, N. Seiberg and E. Witten, "Phases of $\mathrm{N}=1$ supersymmetric gauge theories and matrices," JHEP 0302, 042 (2003) arXiv:hep-th/0301006.

[6] F. Cachazo, N. Seiberg and E. Witten, "Chiral Rings and Phases of Supersymmetric Gauge Theories," JHEP 0304, 018 (2003) arXiv:hep-th/0303207.

[7] F. Ferrari, "On exact superpotentials in confining vacua," Nucl. Phys. B 648, 161 (2003) arXiv:hep-th/0210135.

[8] F. Ferrari, "Quantum parameter space and double scaling limits in N = 1 super YangMills theory," Phys. Rev. D 67, 085013 (2003) arXiv:hep-th/0211069.

[9] F. Ferrari, "Quantum parameter space in super Yang-Mills. II," Phys. Lett. B 557, 290 (2003) arXiv:hep-th/0301157.

[10] R. Dijkgraaf, M. T. Grisaru, C. S. Lam, C. Vafa and D. Zanon, "Perturbative computation of glueball superpotentials," arXiv:hep-th/0211017.

[11] G. Veneziano and S. Yankielowicz, "An Effective Lagrangian For The Pure N=1 Supersymmetric Yang-Mills Theory," Phys. Lett. B 113, 231 (1982).

[12] F. Cachazo and C. Vafa, "N=1 and $N=2$ geometry from fluxes," arXiv:hepth/0206017.

[13] F. Cachazo, K. A. Intriligator and C. Vafa, "A large N duality via a geometric transition," Nucl. Phys. B 603, 3 (2001) arXiv:hep-th/0103067.

[14] N. Seiberg and E. Witten, "Electric - magnetic duality, monopole condensation, and confinement in N=2 supersymmetric Yang-Mills theory," Nucl. Phys. B 426, 19 (1994) [Erratum-ibid. B 430, 485 (1994)] arXiv:hep-th/9407087.

[15] N. Seiberg and E. Witten, "Monopoles, duality and chiral symmetry breaking in $\mathrm{N}=2$ supersymmetric QCD," Nucl. Phys. B 431, 484 (1994) arXiv:hep-th/9408099].

[16] P. C. Argyres and A. E. Faraggi, "The vacuum structure and spectrum of N=2 supersymmetric SU(n) gauge theory," Phys. Rev. Lett. 74, 3931 (1995) arXiv:hepth/9411057].

[17] A. Klemm, W. Lerche, S. Yankielowicz and S. Theisen, "Simple singularities and N=2 supersymmetric Yang-Mills theory," Phys. Lett. B 344, 169 (1995) arXiv:hepth/9411048]. 
[18] A. Hanany and Y. Oz, "On the quantum moduli space of vacua of $\mathrm{N}=2$ supersymmetric SU(N(c)) gauge theories," Nucl. Phys. B 452, 283 (1995) arXiv:hep-th/9505075.

[19] T. Eguchi and Y. Sugawara, "Branches of $N=1$ vacua and Argyres-Douglas points," JHEP 0305, 063 (2003) arXiv:hep-th/0305050.

[20] G. Bertoldi, "Matrix models, Argyres-Douglas singularities and double scaling limits," JHEP 0306, 027 (2003) arXiv:hep-th/0305058.

[21] M. R. Douglas and S. H. Shenker, "Dynamics of SU(N) supersymmetric gauge theory," Nucl. Phys. B 447, 271 (1995) arXiv:hep-th/9503163.

[22] P. C. Argyres and M. R. Douglas, "New phenomena in SU(3) supersymmetric gauge theory," Nucl. Phys. B 448, 93 (1995) [arXiv:hep-th/9505062].

[23] P. C. Argyres, M. Ronen Plesser, N. Seiberg and E. Witten, "New N=2 Superconformal Field Theories in Four Dimensions," Nucl. Phys. B 461, 71 (1996) arXiv:hepth/9511154].

[24] T. Eguchi, K. Hori, K. Ito and S. K. Yang, "Study of $N=2$ Superconformal Field Theories in 4 Dimensions," Nucl. Phys. B 471, 430 (1996) [arXiv:hep-th/9603002.

[25] S. Terashima and S. K. Yang, "Confining phase of $\mathrm{N}=1$ supersymmetric gauge theories and N = 2 massless solitons," Phys. Lett. B 391, 107 (1997) arXiv:hep-th/9607151.

[26] S. Terashima and S. K. Yang, "ADE confining phase superpotentials," Nucl. Phys. B 519, 453 (1998) arXiv:hep-th/9706076.

[27] J. de Boer and Y. Oz, Nucl. Phys. B 511, 155 (1998) [arXiv:hep-th/9708044.

[28] A. Gorsky, A. I. Vainshtein and A. Yung, Nucl. Phys. B 584, 197 (2000) arXiv:hepth/0004087. 